Geology, Geophysics \& Environment • 2012 • Vol. 38 • No. 4 • 439-448

http://dx.doi.org/10.7494/geol.2012.38.4.439

\title{
PRACTICAL APPLICATION OF HIGH RESOLUTION GROUND PENETRATING RADAR METHOD INSIDE BUILDINGS
}

\author{
Ewelina MAZUREK \& Mikołaj LYSKOWSKI \\ AGH University of Science and Technology, Faculty of Geology, \\ Geophysics and Environmental Protection; \\ al. Mickiewicza 30, 30-059 Krakow, Poland; \\ e-mail: emazurek@geol.agh.edu.pl,.lyskowski@geol.agh.edu.pl
}

\begin{abstract}
An infrastructure development requires a precise indoor localization plan of cables, pipes or reinforcing elements. Due to the age of many buildings in Poland such plans often do not exist or do not contain the evidence of numerous activities carried out over years like repairs or modifications. Due to this situation demands for application of geophysical methods grow, especially with respect to the Ground Penetrating Radar (GPR) method. A possibility of precise localization of hidden objects arises with the use of high frequency antennas together and with precise measuring grid. Research carried out at the request of one of the departments of AGH University of Science and Technology in building B2 showed great potential of GPR methods in this field. The study was devoted to recognition of structure of the concrete floor. The obtained results gave very precise location of numerous objects of interest and they became a good sample of high resolution investigations. The survey was performed with ProEx unit, produced by a Swedish company - Mala Geoscience, which is in the possession of the Department of Geophysics, Faculty of Geology, Geophysics and Environmental Protection.
\end{abstract}

Key words: GPR, sleeve, high frequency GPR antenna, foundations of buildings, reinforced concrete

\section{INTRODUCTION}

Trying to locate objects placed under the floors and in the walls of buildings without interfering with their structure is often problematic. Sometimes, the system components such as pipes, cables and ducts are not clearly shown, or do not appear at all in the building 
plans. Similar problems occur when work must be done on reinforced concrete elements, in that case drilling through their steel components should be avoided. Location of such facilities is possible by the use of Ground Penetrating Radar method (GPR). This method gives excellent results and enables to determine the location of the searched objects with high precision.

\section{AREA OF THE STUDY}

The study was performed on behalf of the Faculty of Mechanical Engineering and Robotics placed in the pavilion B2 in the complex of buildings of the AGH University of Science and Technology in Krakow. Due to the nature and location of the objects it was decided that it would be appropriate to apply the GPR method. The main aim of this study was to locate the old steel sleeve placed inside the foundation of the building. The place where the investigation was performed was tentatively named the Area A. Currently the objects of interest are under the floor. Because of the planned investments there is a necessity to locate those sleeves and use them as a foundation for the new measuring apparatus.

In order to expand on this case study, additional investigation was performed in the adjacent room - named the Area B. In this case the main aim was to identify the spot where the floor could be drilled to a depth that would allow the attachment of steel scaffolding for stabilization of a new item of heavy laboratory eqiupment. Therefore, in order to suggest the appropriate location precisely it was necessary to identify the exact location of the object embedded in the floor structure. There was also necessary to make sure that the building plans accurately reflect the actual situation, which was especially important for the planned use of the site. As in case of the Area A, the test equipment was identical. Figures 2 and 3 (pp. 444, 445) contain a schematic mini map with marked areas of measurement. Due to the small size of the objects embedded in the floor and the expected limits of depth - searching of steel reinforcement in concrete structures as well as the location of system components, it was decided to use $1600 \mathrm{MHz}$ and $800 \mathrm{MHz}$ shielded, ground coupled antenna. For comparison of the results, the comparative investigations were performed with the use of both antennas in the Area B and along the same profile line in each case. The pre-processing method on site measurements enabled to conclude that that higher frequency antenna is a better choice due to much higher resolution.

The investigation in the Area A consisted of 20 parallel profiles measured with the $1600 \mathrm{MHz}$ antenna, running in straight lines and starting at the same point. The distance between the profiles was $0.2 \mathrm{~m}$. Due to the nature of tests performed inside the building, it was impossible to obtain profiles of equal length. Profiles from 16 to 20 were carried out through the door frame. The lengths and location of the profiles in the Area A are shown at Figure 1. 


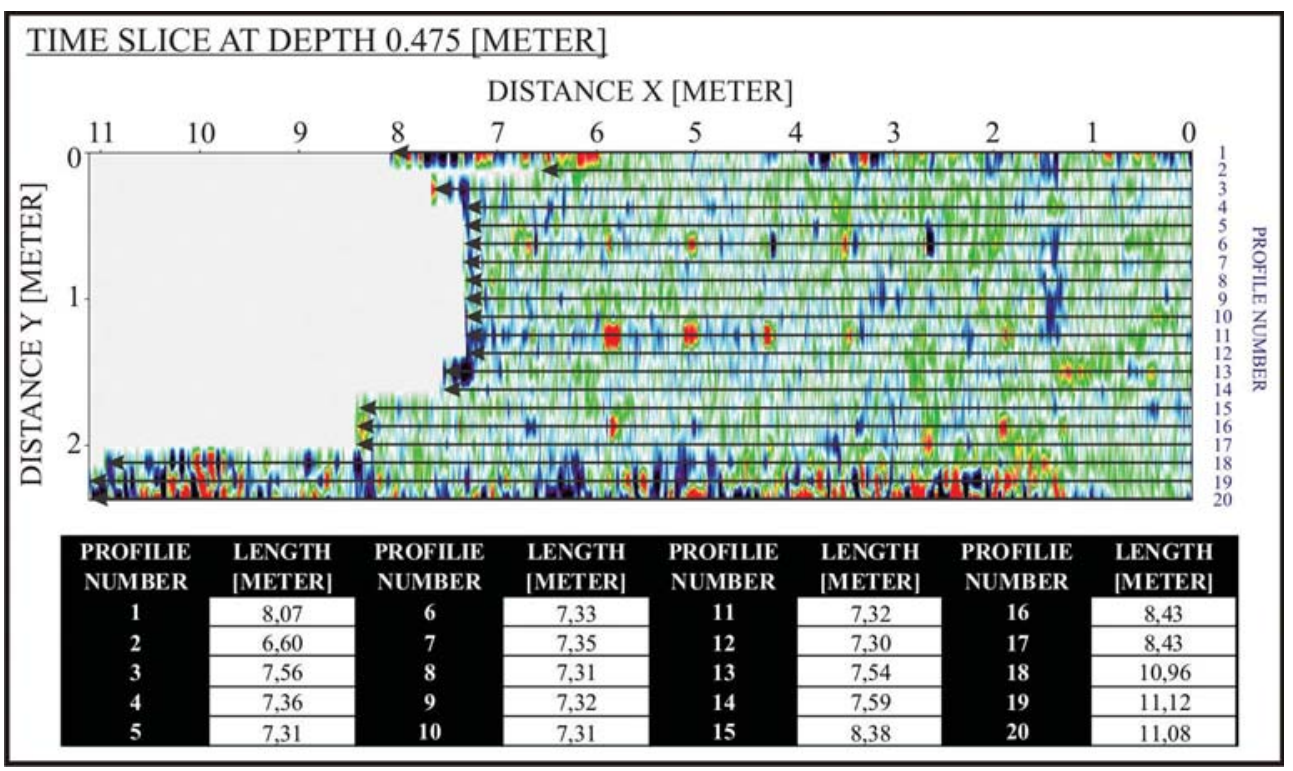

Fig. 1. Map of profiles localization in Area A with table of lengths (time slice at depth $0.475 \mathrm{~m}$ as background)

The Area B included 17 parallel profiles for each of the two types of antennas: $800 \mathrm{MHz}$ and $1600 \mathrm{MHz}$. The profiles run in straight lines with spacing between them at $0.2 \mathrm{~m}$. In this case, due to lack of objects that would hinder measurement, all profiles had the same length - $4.0 \mathrm{~m}$. The measurements in the Area B were performed mainly for comparative test of the two antennas resolution and penetration abilities.

\section{METHOD}

The GPR method is a geophysical method that allows locating objects below the surface of the earth, concrete, water and other medium using electromagnetic waves (Karczewski et al. 2011). The electromagnetic waves are sent into the medium by antennas. On the border of two media having different electrical properties there occur the phenomena of refraction and reflection, while absorption occurs inside layers (Karczewski et al. 2011). The amplitude of the reflected electromagnetic pulse is proportional to the reflection coefficient on the border of the different media. With the increasing difference in the dielectric constant, the reflectivity increases, which translates into an increase in the amplitude of the pulse (Misiewicz 2006).

The simplest GPR system consists of a transmitting antenna, a transmitter, a receiver unit with a separate antenna for receiving waves and the trigger distance electronics. Due to the structure of the antennas they can be divided into two different types: shielded 
and unshielded. In the shielded type, the transmitting and receiving antennas are located in an electromagnetic housing shield, making them less susceptible to external interference such as reflections from trees. Unshielded antennas are not physically integrated and are not shielded from the interference. Because of separation between the transmitting and receiving antennas, there is a possibility to carry out investigations with different mutual spacing distance between the dipoles, or with different polarisations of the dipoles in relation to the profile (Ortyl 2006). The main processing unit (called CPU) synchronizes the clocks of the transmitter and the receiver. Transmitter sends a pulse to the transmitting antenna with command to generate the electromagnetic wave. The waves reflected at the boundary between geological layers arrive to the receiving antenna where the receiver records the time course of the return signal. The CPU controls the data acquisition and stores it in the computer memory in real time (Karczewski et al. 2011).

Today, the GPR systems use the antennas with frequencies from $10 \mathrm{MHz}$ to even 6,000 MHz (British custom GPR system Groundvue 5C). The frequency of the emitted signal has a decisive influence on both the resolution and the effective penetration depth (Karczewski et al. 2011). The higher the frequency, the shorter the depth range and higher the resolution. Thus, through the use of high frequency aerials it is possible to detect smaller objects, but at shallower depths. The measurement results are provided in a form of the echograms. These sections are kind of geological slices in which the horizontal axis is a profile distance, while the vertical axis shows the time of wave propagation. To convert time into depth it is necessary to determine the average velocity of propagation of the wave - this approximate procedure rescales the vertical time axis into the axis of depth (Karczewski et al. 2011).

It is necessary to mention some kind of capriciousness of the GPR method. For unknown reasons, the field measurements in seemingly ideal conditions for application of this method - for example dry bedrock - it may happen that the obtained results using the GPR will be almost uninterpretable. On the other hand, the locality which is strongly saline or very damp can give very clear and easily interpretable results (Łyskowski \& Wardas 2012). The results of such kind were obtained in the experimental studies in the Wieliczka Salt Mine on the water-saturated shaft wall.

The last and necessary to mention is the coefficient of a wave attenuation, which has decisive influence on the depth range of the GPR method. The propagation of the electromagnetic waves accompanied by attenuation - a gradual reduction of an electromagnetic pulse amplitude along the travel path.

The attenuation of the electromagnetic wave in a medium depends on four basic factors:

1) geometrical divergence of the wave front corresponding to point source of the wave;

2) scattering related to inhomogeneity of the medium (spatial variations of transmissivity, granulation or layering) (Annan 2001);

3) frequency dependent propagation dispersion of the waves;

4) electrical conductivity of the medium, usually corresponding to presence of electrolytes in pore spaces. 
A decisive role in the selection of the appropriate antenna is played by a parameter called resolution. The concept for the GPR method is more complex. Here are cases of vertical and horizontal resolution. Each of them is described by a different model and its value depends on other factors (Karczewski et al. 2011).

We should start with a question what is the resolution of the GPR method. It is the minimum distance $\Delta R$ between two objects so that they are visible on the echogram as separate items. Only a descriptive definition of resolution is insufficient, no figure can properly visualize it. Noon (1998) defines the vertical resolution as: $\Delta R_{\text {vertical }}=\frac{1}{2} \cdot \lambda_{C}$, where $\lambda_{C}$ is local electromagnetic wavelength (in the medium) which corresponds to the central frequency of the antenna.

Assuming that $r \gg \Delta R_{\text {vertical }}$ we may describe the horizontal resolution. In practice, it depends on the depth of the objects and the antenna frequency: $\Delta R_{\text {horizontal }}=\sqrt{\frac{r \cdot \lambda_{C}}{2}}$, where $r$ is the depth of the disturbing bodies (Noon 1998). These formulas tries to estimate limits of resolution, but usually another factors are responsible for low clarity of echograms.

\section{RESULTS}

Prior to presentation of the results of the GPR measurements it is necessary to process the raw data. For this purpose a computer program ReflexW, version 5.5 and the procedure with the following steps were employed (using the original procedures names):

- "movestarttime" - hand typed, fixed value of time (in nanoseconds [ns]), which is the time of the first signal occurrence in the given scan;

- "subtract-DC-shift" - the program calculates mean value ("DC-value") for the given time range of the given scan and subtracts this value from all data values of the scan (ReflexW Manual);

- "background removal" - program creates mean trace and subtracts this trace from each trace (along X-axis) (ReflexW Manual);

- "substract-mean (dewow)" - creates within the moving time window (along Z-axis) a mean value and subtracts the value from the actual data value (ReflexW Manual);

- "gain function" - strengthening time-dependent multiplayer applied to enhance the farther reflexes;

- "time slice (3D)" - creates a slice in the vertical axis.

The procesing sequence presented above was chosen as the most effective among several that have been used.

The results for Area A are summarized in Figure 2 and for Area B in Figure 3. The interpretation and the discussion are given in the penultimate chapter of this paper. 


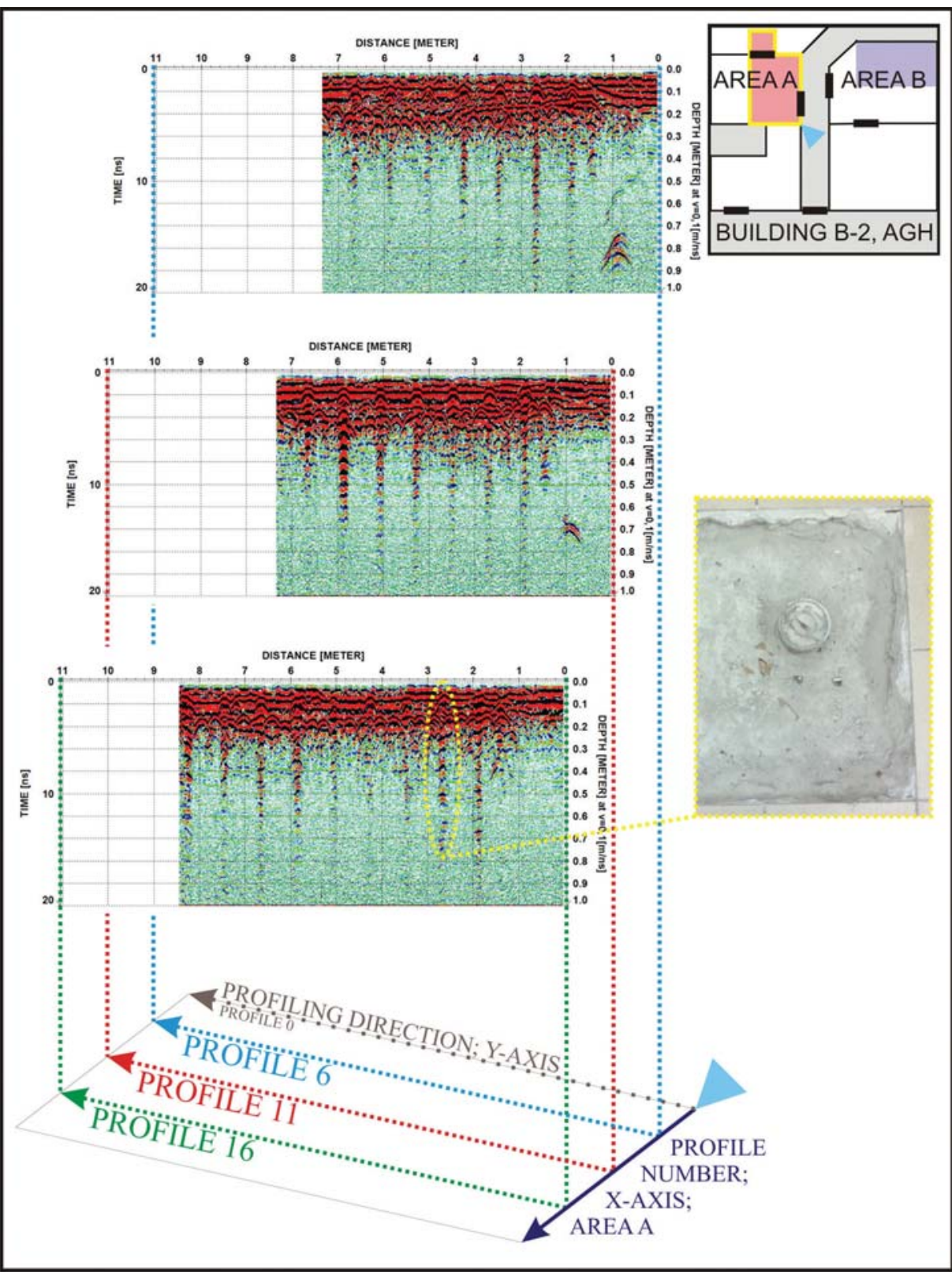

Fig. 2. Three vertical cross-sections along $\mathrm{X}$-axis with visible anomalies corresponding to steel sleeves in Area A over a schematic plane of profiling and the photo of one unveiled sleeve (figure also contains mini map with lighted investigation place on a schematic plan of the building B-2). For construction of the depth scale of the vertical Z-axis, the velocity $0.1 \mathrm{~m} / \mathrm{ns}(\varepsilon=9)$ was assumed 


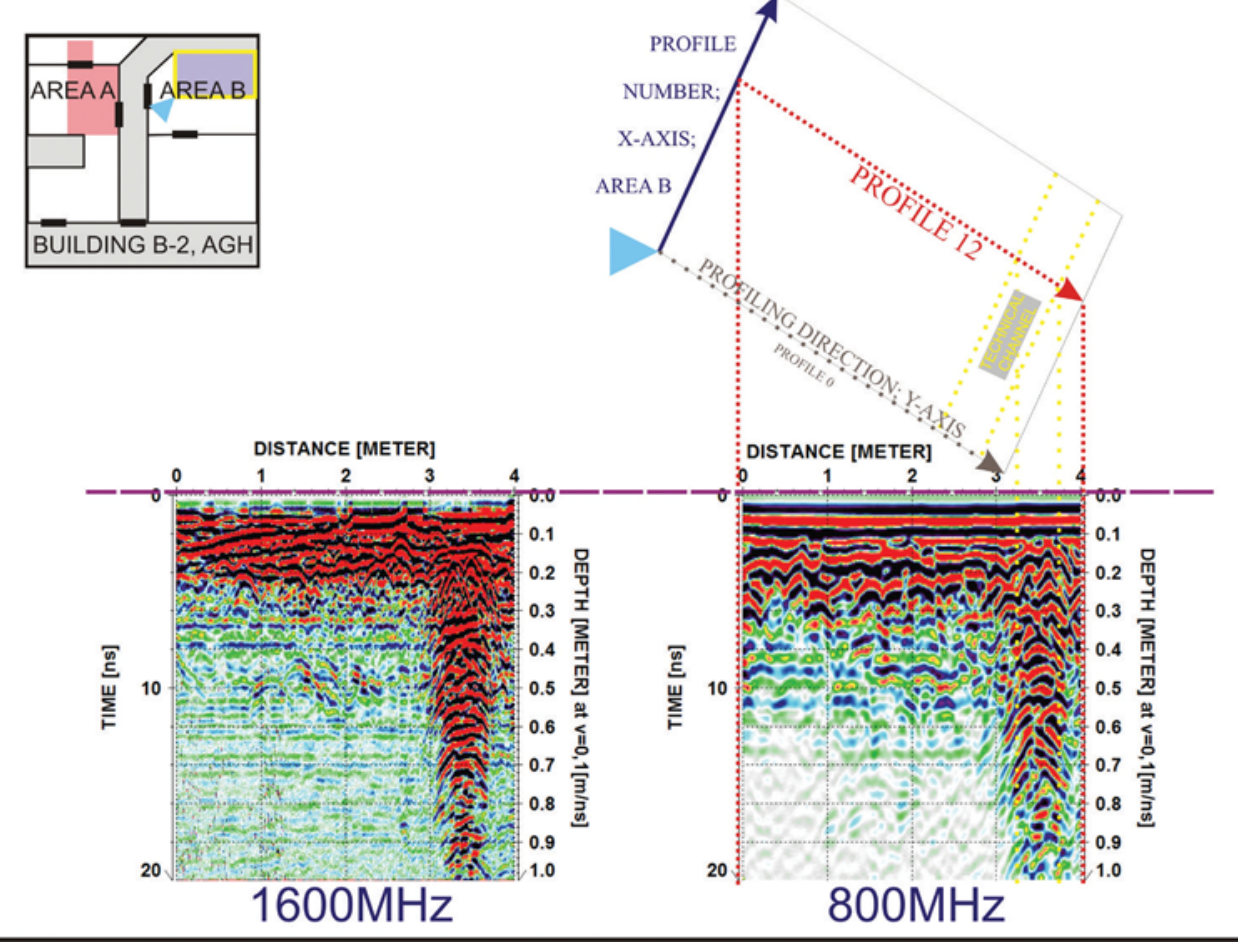

Fig. 3. Comparison of profile 12 in Area B made with usage of two antennas: $800 \mathrm{MHz}$ and $1,600 \mathrm{MHz}$ with schematic plane of profiling (figure also contains mini map with lighted investigation place on a schematic plan of the building B-2). For construction of the depth scale of the vertical Z-axis, the velocity $0.1 \mathrm{~m} / \mathrm{ns}(\varepsilon=9)$ was assumed

Figure 2 shows three echograms with visible anomalies. The source position for those parabolic effects on X-Y plane have lateral continuation (in X direction) and are arranged in a parallel line system what may be well illustrated by systematic survey of echograms and slices. Each of 22 iron sleeves occurs at a depth 0.08 meter below floor surface level. Depth was read assuming the wave propagation velocity at $0.1 \mathrm{~m} / \mathrm{ns}(\varepsilon=9)$.

Interpretation of the echograms obtained as a result of processing in the case of the Area B (Fig. 3) enabled to recognize the location of a technical channel with visible diffractions from cables at depth of about $0.2 \mathrm{~m}$ (the anomaly appears at the end of the echogram) and a system of steel reinforcement elements (the visible hyperbolae starting at a distance of 0.8 meter of the profile, and their peaks occur at a depth from $0.1 \mathrm{~m}$ to $0.25 \mathrm{~m}$ ). There is also an inter-layer horizon visible under the reinforcement system at a distance $0.8-2.5 \mathrm{~m}$ and depth $0.4-0.55 \mathrm{~m}$. We can easily indicate the beginning of it at the technical channel. This anomaly is invisible and blends with another signals on the echogram made with $800 \mathrm{MHz}$ antenna. Depth was read assuming the wave propagation velocity at $0.1 \mathrm{~m} / \mathrm{ns}(\varepsilon=9)$. 


\section{DISCUSSION}

The application of two shielded antennas of different frequencies at a reconnaissance stage of this study demonstrated that the $1,600 \mathrm{MHz}$ antenna produced results of better clarity and resolution.

The final results of 20 echograms from research in the Area A, after careful analysis showed that the horizontal slices (constant time slices) or vertical cross-sections along the profiles direction (X-axis) are the best way for visualization of the results.

The echograms analysis in form of 3D cube showed that 22 steel sleeves can be found in the investigated area. To confirm the survey results, one such sleeve was exposed at the spot in the Area A shown in Figure 2. The sleeves are detectable thanks to generation of strong dispersed signal, which is related to the high conductivity of steel.

The results from Area B indicate that the measurements performed with 1,600 MHz antenna are more legible and contain a lot more details of the construction of this test facility. Echogram reveals the reinforcement in the concrete, which is invisible in the $800 \mathrm{MHz}$ antenna image. The image of the technical channel is also more detailed. The inter-layer horizon does not appear clearly at the echogram obtained with the $800 \mathrm{MHz}$ antenna, but is shown as some raw disturbances. On the other hand the effective penetration of the $800 \mathrm{MHz}$ antenna is about 1.5 times deeper than $1,600 \mathrm{MHz}$ antenna.

In the interpretation of the echograms the Authors kept in mind that the medium is not homogeneous and the assumed wave propagation velocity would be averaged. This situation may cause inaccuracies in determining the depth of the disturbing bodies - the so-called "time shift". In the selection of appropriate wave velocity Authors used the available information and selected tabular value assigned to medium built of concrete - wave propagation velocity at $0.1 \mathrm{~m} / \mathrm{ns}(\varepsilon=9)$. The value of dielectric constant for dry concrete ranges between 4 and 9 . The value 9 assumed by the Authors allowed for a very accurate time-to-depth conversion, which has been later confirmed by the depth of the exposed sleeve (about $0.08 \mathrm{~m}$ ).

\section{CONCLUSION}

- In terms of high resolution of the GPR technique as a geophysical method the best results can be obtained with high frequency antennas, which are characterized by a high vertical and horizontal resolution, but shallow effective depth penetration.

- When considering survey in buildings, it is necessary to use non-invasive, quick and compact apparatus method - which perfectly matches the GPR method.

- The 1,600 MHz antenna was a perfect choice for our study of the concrete plate construction. The results obtained by the use of this antenna and the appropriate processing, were more clear and allowed more precise interpretation. 
- In the Area A there were found 22 steel sleeves. Survey in the Area B, in the adjacent room, enabled us to locate the course of a technical channel and the concrete reinforcement system. Also in this area there is an anomaly below the concrete floor, which probably corresponds to some remnants of the older foundations.

\section{REFERENCES}

Annan A.P., 2001. Ground Penetrating Radar Workshop Notes. Sensors \& Software, Ontario.

Karczewski J., Ortyl Ł. \& Pasternak M., 2011. Zarys metody georadarowej. Wydanie 2 poprawione $i$ rozszerzone. Uczelniane Wydawnictwa Naukowo-Dydaktyczne AGH, Kraków.

Łyskowski M. \& Wardas-Lasoń M., 2012. Georadar investigations and geochemical analysis in contemporary archeological studies. Geology, Geophysics \& Environment, 38, 3, 2012, 307-315.

Misiewicz K., 2006. Geofizyka archeologiczna. Instytut Archeologii i Etnologii Polskiej Akademii Nauk, Warszawa.

Noon D.A., Stickley G.F. \& Longstaff D., 1998. A frequency-independent characterization of GPR penetration and resolution performance. Journal of Applied Geophysics, 40 (1-3), 1998, 127-137.

Ortyl Ł., 2006. Badanie przydatności metody georadarowej w geodezyjnej inwentaryzacji struktur i obiektów podpowierzchniowych. Wydział Geodezji Górniczej i Inżynierii Środowiska AGH, Kraków [Ph.D. Thesis, unpublished].

ReflexW Manual, 2009. User Guide, SandmeierGeo. Karlsruhe, Germany. 
